\title{
Information de la Commission technique de lutte contre le dopage (CLD)
}

\author{
M. Kamber ${ }^{a}$, N. Mahler $^{a}$, M. Strupler ${ }^{b}$
}

\section{Nouvelle Liste des interdictions à compter du 1er $^{\text {janvier }} 2005$}

Une nouvelle liste des substances et méthodes dopantes interdites entrera en vigueur le $1^{\mathrm{er}}$ janvier 2005. C'est la deuxième fois que l'Agence mondiale antidopage (AMA) assume seule la responsabilité de son contenu. Selon le Code antidopage de l'AMA et le Standard international pour l'élaboration de la liste des produits interdits, seules les substances et les méthodes remplissant deux des trois critères suivants doivent figurer dans cette liste:

- les connaissances médicales et scientifiques actuelles attestent que la substance ou la méthode est à même d'améliorer la performance sportive;

- les connaissances médicales et scientifiques actuelles attestent que la substance ou la méthode présente un risque réel ou potentiel pour la santé;

- l'AMA estime que l'utilisation de la substance ou de la méthode porte atteinte à l'éthique sportive (esprit du sport).

Les organisations partenaires intéressées ont été invitées à prendre position sur la première mouture de la Liste des interdictions 2005 dans le cadre d'une procédure de consultation menée durant l'été 2004. La Commission technique de lutte contre le dopage (CLD) a profité de cette occasion pour faire un certain nombre de suggestions et pour clarifier certains points. Elle a ainsi demandé des éclaircissements sur certaines formulations, p.ex. concernant l'adrénaline, l'interdiction des perfusions intraveineuses ou le listage de certaines substances. La CLD a notamment proposé de n'interdire l'usage des glucocorticoïdes que dans certaines disciplines, et donc de faire passer ces agents dans la catégorie des «substances interdites dans certains sports»; elle a par ailleurs suggéré de n'interdire la prise de cannabinoïdes que dans certains sports, voire de les rayer purement et simplement de la liste. Si l'AMA a tenu compte de certaines remarques, elle n'a malheureusement pas donné son avis sur les propositions qui lui ont été soumises, ni justifié ou expliqué ses décisions.

\section{Nouvelle structure de la Liste des interdictions}

La Liste des interdictions a été remodelée. La liste valable à compter du $1^{\text {er }}$ janvier 2005 débutera par les substances et les méthodes interdites en permanence, que ce soit en compétition ou hors compétition. Si le nombre des classes de substance et des méthodes interdites demeure inchangé par rapport à 2004, l'ordre de présentation a, lui, été modifié.

Comme dans la liste de 2004, seules certaines valeurs limites seront indiquées dans la liste de 2005 (p. ex. pour l'éphédrine, la cathine et le salbutamol). En revanche, d'autres valeurs limites (p.ex. pour les cannabinoïdes, la morphine et la nandrolone) ne figureront plus que dans les documents techniques destinés aux laboratoires et ce, pour que l'on ne puisse pas penser que l'usage de ces substances soit autorisé dans une certaine mesure. Cette solution est toutefois loin d'être optimale en termes de transparence.

Pour certaines classes de substances dont la liste d'exemples n'est pas exhaustive, le qualificatif «biologique» remplace le qualificatif «pharmacologique».

\section{Modifications relatives aux substances interdites}

\section{Agents anabolisants}

Les neuf stéroïdes anabolisants androgènes (SAA) suivants figurent dans la catégorie des SAA exogènes:18 $\alpha$-homo-17 $\beta$-hydroxyestr-4-en-3-one, calustérone, delta1-androstènediol, éthylestrénol, furazabol, méthyldiénolone, méthyltriénolone, norclostébol et tétrahydrogestrinone. Une autre catégorie regroupant de nombreux métabolites et isomères de stéroïdes endogènes (c'està-dire produits par l'organisme) a par ailleurs été ajoutée à la liste.

La liste des SAA endogènes est désormais clôturée.

Contrairement à ce qui était indiqué dans la version de juillet 2004 mise en consultation, il est maintenant obligatoire de procéder à des investigations complémentaires lorsque le rapport testostérone / épitestostérone est de 4 pour 1 (contre 6 pour 1 auparavant). Cette disposition incom- 
préhensible augmentera considérablement les charges administratives et financières de la CLD.

Le zilpatérol est désormais inclus dans la catégorie des «autres agents anabolisants».

\section{Hormones et substances apparentées}

Cette classe de substances, appelée «hormones peptidiques» en 2004, a été rebaptisée. Le principal changement est le suivant: les substances qui n'étaient jusqu'alors interdites que chez les athlètes de sexe masculin, telles que les gonadotrophines (p.ex. hCG), sont désormais interdites chez tous les athlètes. Il reste encore à déterminer avec précision comment et à qui communiquer un résultat d'analyse «positif». A nos yeux en effet, une éventuelle grossesse fait partie des données personnelles particulièrement sensibles.

Autres nouveautés: les facteurs de croissance mécanique (MGFs) ont été ajoutés comme exemple dans la sous-catégorie des hormones de croissance, et les deux sous-catégories de gonadotrophines ont été fusionnées.

\section{Bêta-2 agonistes}

Tous les bêta- 2 agonistes sont désormais interdits en et hors compétition. Comme en 2004, seules quatre substances peuvent être utilisées en inhalation moyennant une autorisation d'usage à des fins thérapeutiques (AUT) abrégée: le formotérol, le salbutamol, le salmétérol et la terbutaline. Il ne faut pas s'attendre à des charges administratives supplémentaires car la plupart des demandes d'AUT pour les bêta- 2 agonistes ne concernaient déjà pas exclusivement les compétitions.

\section{Agents avec activité anti-østrogène}

Cette catégorie a été divisée en 3 sous-catégories. Les substances suivantes sont désormais citées comme exemples: l'anastrozole, le létrozole, l'aminogluthémide, l'exémestane, le formestan, la testolactone, le raloxifène, le torémifène et le fulvestrant. En outre, les substances qui n'étaient jusqu'à présent interdites que chez les athlètes de sexe masculin sont désormais prohibées chez tous les athlètes, sans distinction de sexe.

\section{Diurétiques et autres agents masquants}

Le titre de cette catégorie a été modifié. La métolazone a été ajoutée comme exemple de diurétique, tandis que le mersalyl a été supprimé. Les inhibiteurs de l'alpha-réductase (p.ex. finastéride et dutastéride) ont par ailleurs été ajoutés à la liste des agents masquants interdits.

\section{Stimulants}

La famprofazone et la fencamine ont été ajoutées à la liste d'exemples. Une note précisant le statut de l'adrénaline a également été ajoutée: l'adrénaline associée à des agents anesthésiques locaux, ou utilisée sous forme de préparation à usage local (p. ex. par voie nasale ou ophtalmologique), n'est pas interdite. Le bupropion a en outre été intégré au Programme de surveillance 2005.

\section{Narcotiques}

Le fentanyl et ses dérivés ont été ajoutés à la liste des substances interdites dans cette catégorie (liste clôturée).

\section{Cannabinoïdes}

Rien n'a changé par rapport à la liste de 2004: l'usage de cannabinoïdes demeure interdit en compétition. En 2004, plusieurs cas de dopage au cannabis ont été recensés en Suisse. Certains athlètes incriminés avaient avancé comme argument qu'ils avaient «fumé passivement». Or une athlète qui avait fait appel en première instance devant le Tribunal arbitral du sport (TAS) au motif de l'inhalation passive a été déboutée par ledit tribunal. C'est pourquoi la CLD conseille aux athlètes de renoncer à toute consommation de cannabinoïdes (cannabis, haschisch, marijuana, etc.) pendant toute la durée de leur saison sportive. Elle les met également en garde contre le danger de l'inhalation passive (séjour prolongé dans de petites pièces très enfumées et mal aérées).

\section{Glucocorticoïdes}

L'année 2004 a été marquée par un gros travail administratif dû aux nombreuses demandes d'autorisations d'usage à des fins thérapeutiques (AUT). Cette charge de travail risque de diminuer un peu en 2005 dans la mesure où les préparations cutanées à base de glucocorticoïdes seront de nouveau autorisées (plus d'AUT requise). L'administration locale, topique et intra-articulaire de glucocorticoïdes en compétition requiert l'obtention d'une AUT abrégée. Leur administration systémique (par voie orale ou intramusculaire) continue d'être interdite, et requiert donc obligatoirement l'obtention d'une AUT standard auprès du Comité pour l'autorisation d'usage à des fins thérapeutiques (CAUT).

\section{Modifications relatives aux méthodes interdites}

\section{Manipulation chimique et physique}

Le qualificatif «pharmacologique» a été supprimé du titre car les substances correspondantes sont maintenant répertoriées dans la catégorie des diurétiques et autres agents masquants. 
L'AMA interdit désormais les perfusions intraveineuses comme méthode dopante, excepté dans le cadre légitime d'un traitement médical aigu. Renseignements pris auprès de l'AMA, il apparaît que cette interdiction ne vise pas à mettre les médecins sous tutelle et qu'elle concerne principalement les perfusions aux fins d'hémodilution. Cette mesure poursuit en outre un objectif éducatif, puisqu'elle doit sensibiliser l'opinion au problème des perfusions non justifiées sur le plan médical utilisées en milieu sportif. Elle doit également protéger les athlètes dans la mesure où il est relativement facile de leur administrer des produits dopants à leur insu par perfusion. Les représentants de l'AMA ont promis de fournir toutes les précisions nécessaires aux personnes concernées.

\section{Groupe cible d'athlètes soumis à des contrôles}

Selon le Code antidopage de l'AMA et le statut de Swiss Olympic concernant le dopage, qui a été adapté en conséquence, des groupes cibles nationaux et internationaux d'athlètes soumis à des contrôles antidopage doivent être constitués. De manière générale, les dispositions antidopage s'appliquent à tous les athlètes. Seules les exigences administratives varient en fonction de l'appartenance ou non à un groupe cible. Tous les athlètes de haut niveau, nationaux ou internationaux, qui ont été inclus dans un groupe cible national ou international sont informés de leur appartenance par leur fédération sportive. Ils sont tenus de communiquer leur lieu de séjour et sont soumis à certaines conditions en cas de retrait (décision de rester dans le groupe cible ou de le quitter) et aux dispositions en vigueur pour les AUT (obligation de déposer au préalable une demande d'AUT). Les autres athlètes peuvent demander une AUT mais n'y sont pas tenus. La CLD peut en outre exiger rétrospectivement l'AUT en cas d'analyse positive ou de besoin.

\section{Nouvelle présentation du site Internet}

Le site www.dopinginfo.ch est très apprécié des utilisateurs, qui sont de plus en plus nombreux à le visiter. Il a été récemment adapté afin de répondre encore mieux aux besoins des différents groupes d'utilisateurs. La nouvelle version sera progressivement mise en ligne à partir du début de l'année 2005. La CLD a en outre décidé d'en faire le site officiel de lutte contre le dopage en Suisse. La nouvelle Liste des interdictions et les formules officielles peuvent être téléchargés de ce site. Ceci est la raison pour laquelle la Liste des interdictions n'accompagne plus le Bulletin des médecins suisses. 